\title{
Spectacular flyover of Mars
}

Mars Express video shows near-complete topographical map of planet's surface - and sets it to music.

\section{Elizabeth Gibney}

28 October 2013

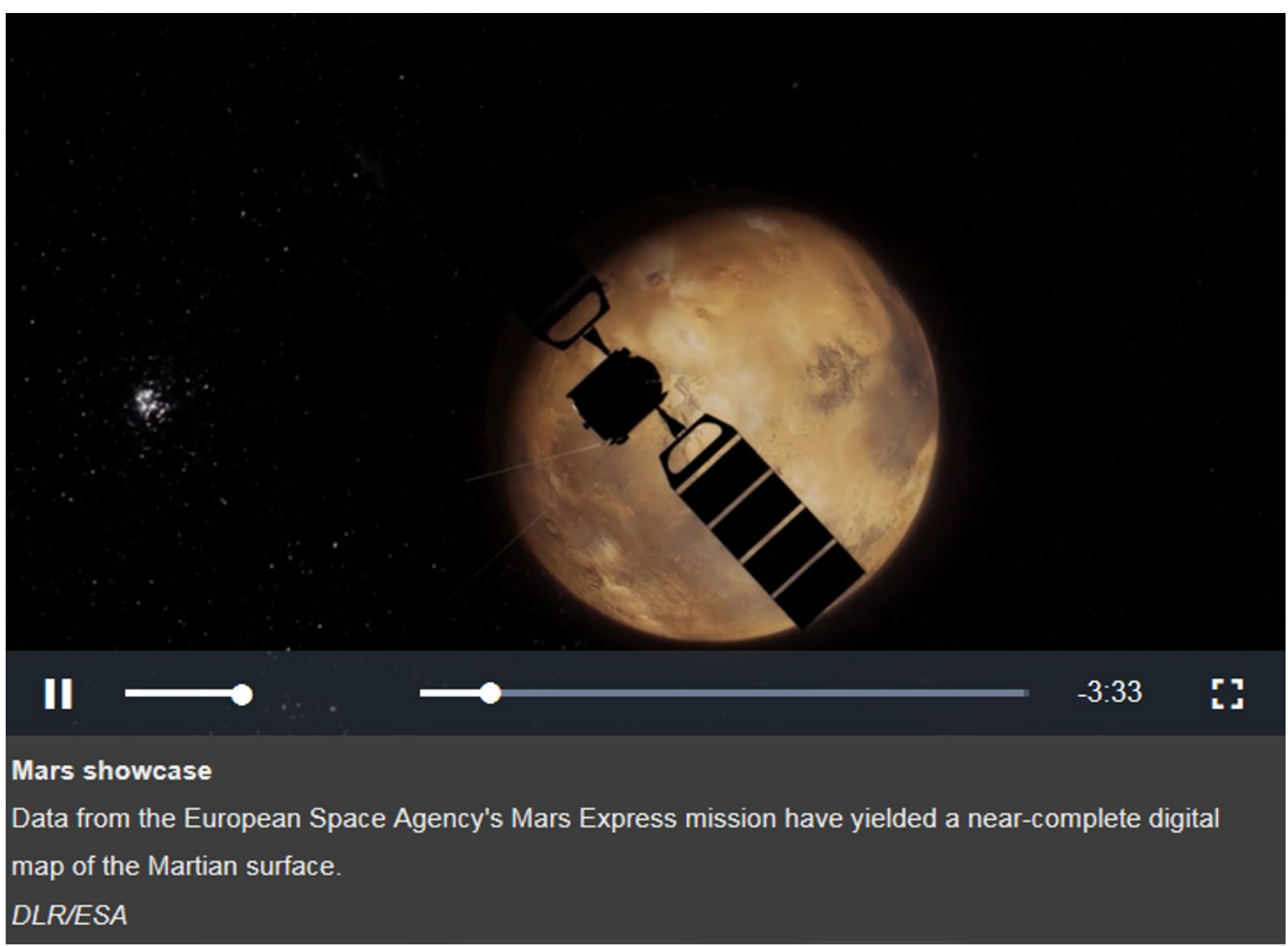

A video based on topographical data of Mars taken by a European satellite gives Earth-dwellers an aerial view of the red planet's surface.

The mountains, craters, ancient river beds and lava flows that mark the Martian landscape are visible in images from a stereographic camera aboard the European Space Agency's Mars Express probe.

ESA released the video, which was produced by German Aerospace Center (DLR) of Cologne, Germany, on 28 October as part of celebrations commemorating the tenth anniversary of the Mars Express launch, in June 2003. The DLR's Stephan Elgner, a member of the mission's planetary cartography team, wrote the original soundtrack.

Mars Express has so far orbited the planet nearly 12,500 times, building up an almost planet-wide digital topographical model.

The spacecraft has outlived its initial mission of one Martian year — which corresponds to roughly two Earth years - and is now expected to continue orbiting the planet until the end of 2014 .

Nature | doi:10.1038/nature.2013.14041 